\title{
From Nanotechnology to Nanoarchitectonics
}

\author{
Katsuhiko Ariga
}

Received: 9 January 2015 / Accepted: 10 January 2015/Published online: 29 January 2015

(C) Springer Science+Business Media New York 2015

Nanotechnology is undoubtedly one of the key concepts in current science and technology. Since Dr. Richard Feynman [1] said, "There's Plenty of Room at the Bottom," attention, curiosity, and efforts on fabricating ever smaller objects have been paid attention and have been rapidly growing in this decade. Developments of nanotechnology depend considerably on the development of observation technology such as invention of various advanced microscopies. We can now see individual atoms and molecules that can be also manipulated with advanced tools and techniques. These developments create fantastic opportunities in nanoscience. Phenomena at the nanoscale are being continuously discovered. However, we come to know that control of nanoscopic objects is not as easy as we expected. In nanoscopic worlds, these tiny objects have significant influence over several factors including thermal statistical fluctuations. In addition, they do not always behave independently and substantially affect one another. Providing inputs do not always create expected outcomes. These situations are totally different from events in macroscopic and simple microscopic worlds where microtechnology ensures expected causes and effects.

How can we control and handle nano-matter. It can be accomplished by thinking of the physical and chemical interactions under highly influential conditions rather than under an ideal one-to-one relation; i.e., for nano-matter, combined and sophisticated interactions are necessary rather than individual behavior. This paradigm shift would be likened to a shift from using single-tool-based

K. Ariga $(\bowtie)$

World Premier International (WPI) Research Center for

Materials Nanoarchitectonics (MANA), National Institute for

Materials Science (NIMS), Tsukuba, Japan

e-mail: ARIGA.Katsuhiko@nims.go.jp manufacturing to total-construction-of-architecture. It is expedient to embark on the next phase of nanotechnology, which is Nanoarchitectonics. This terminology and its concept were first proposed by Dr. Masakazu Aono at the 1st International Symposium on Nanoarchitectonics Using Suprainteractions in 2000 in Tsukuba, Japan [2, 3]. Actually, the 21st century started with Nanoarchitectonics.

In Nanoarchitecåtonic handling of materials and systems, exact design may be less meaningful; and, the harmonization of incomplete composition and ambiguous structures may work more effectively due to uncertainty based on thermal/statistical fluctuations. These components are assembled into functional nanoarchitectures through self-organization, physical/chemical nanomanipulation, field-induced control for ordering/orientation, and their integrations. Peculiar and unexpected properties may be created upon complexity from huge numbers of component nano-objects. Because this concept is so general, application of the nanoarchitectoncs concept has been recently proposed from materials science [4-6] to biomedical applications [7-9].

Nanotechnology has matured since 1959. In contrast, nanoarchitectonics, which was born in 2000, is still a young child with open questions that are seeking a future destiny. Lots of experiences and experiments in many research fields make nanotechnology a wonderful research endeavor. The use and growth of Nanoarchitectonics in many and increasing laboratories are now necessary.

\section{References}

1. American Physical Society Meeting in 1959

2. M. Aono, Sci. Technol. Adv. Mater. 12, 040301 (2011) 
3. K. Ariga, Q. Ji, J.P. Hill, Y. Bando, M. Aono, NPG Asia Mater. 4, e17 (2014)

4. K. Ariga, A. Vinu, Y. Yamauchi, Q. Ji, J.P. Hill, Bull. Chem. Soc. Jpn. 85, 1 (2012)

5. M. Ramanathan, L.K. Shrestha, T. Mori, Q. Ji, J.P. Hill, K. Ariga, Phys. Chem. Chem. Phys. 15, 10580 (2013)

6. K. Ariga, Y. Yamauchi, G. Rydzek, Q. Ji, Y. Yonamine, K.C.-W. Wu, J.P. Hill, Chem. Lett. 43, 36 (2014)
7. K. Ariga, Q. Ji, T. Mori, M. Naito, Y. Yamauchi, H. Abe, J.P. Hill, Chem. Soc. Rev. 42, 6322 (2013)

8. W. Nakanishi, K. Minami, L.K. Shrestha, Q. Ji, J.P. Hill, K. Ariga, Nano Today 9, 378 (2014)

9. K. Ariga, K. Kawakami, M. Ebara, Y. Kotsuchibashi, Q. Ji, J.P. Hill, New J. Chem. 38, 5149 (2014) 